

anciennement Séminaire de Théorie des Nombres de Bordeaux

Takayuki MORISAWA et Ryotaro OKAZAKI

\title{
Height and Weber's Class Number Problem
}

Tome 28, no 3 (2016), p. 811-828.

<http://jtnb.cedram.org/item?id=JTNB_2016__28_3_811_0>

(C) Société Arithmétique de Bordeaux, 2016, tous droits réservés.

L'accès aux articles de la revue «Journal de Théorie des Nombres de Bordeaux » (http://jtnb.cedram.org/), implique l'accord avec les conditions générales d'utilisation (http://jtnb.cedram. org/legal/). Toute reproduction en tout ou partie de cet article sous quelque forme que ce soit pour tout usage autre que l'utilisation à fin strictement personnelle du copiste est constitutive d'une infraction pénale. Toute copie ou impression de ce fichier doit contenir la présente mention de copyright.

\section{cedram}


Journal de Théorie des Nombres

de Bordeaux 28 (2016), 811-828

\title{
Height and Weber's Class Number Problem
}

\author{
par TAKAYUKI MORISAWA et RYOTARO OKAZAKI
}

\begin{abstract}
RÉSumÉ. Nous étudions la non divisibilité par un nombre premier $\ell$ du nombre de classes $h_{n}$ du $n$-ième étage $\mathbb{B}_{n}$ de la $\mathbb{Z}_{p^{-}}$ extension cyclotomique de $\mathbb{Q}$, où $p$ est un nombre premier fixé. Posons $q=4$ si $p=2$ et $q=p$ si $p \geq 3$ et notons $D(p, s, f)$ l'ensemble des nombres premiers $\ell$ dont l'ordre modulo $q$ vaut $f$ et dont $p^{s}$ est la plus grande puissance de $p$ divisant $\ell^{f}-1$. Dans cet article nous définissons une constante explicite $G(p, s, f)$ ayant la propriété que chaque $h_{n}$ est non divisible par les $\ell$ dans $D(p, s, f)$ tels que $\ell>G(p, s, f)$.
\end{abstract}

Abstract. We discuss indivisibility by prime numbers $\ell$ of the class number of the $n$-th layer $\mathbb{B}_{n}$ of the cyclotomic $\mathbb{Z}_{p}$-extension of $\mathbb{Q}$ where $p$ is an arbitrary fixed prime number.

We denote by $h_{n}$ the class number of $\mathbb{B}_{n}$. Put $q=4$ if $p=2$ or $q=p$ if $p \geq 3$. For positive integers $f$ and $s$, let $D(p, s, f)$ be the set of prime numbers $\ell$ satisfying the following two conditions: (1) the order of $\ell$ modulo $q$ is $f$ and (2) $p^{s}$ is the exact power of $p$ dividing $\ell^{f}-1$. In this paper, we define an explicit function $G(p, s, f)$ which depends only on $p, s$ and $f$. We show that $h_{n}$ is indivisible by every prime number $\ell$ in $D(p, s, f)$ with $\ell>G(p, s, f)$ for every non-negative integer $n$.

\section{Introduction}

Let $p$ be a prime number and $\mu_{m}$ the group of all $m$-th roots of unity. We put $q=4$ if $p=2$ or $q=p$ if $p \geq 3$. We denote by $\mathbb{B}_{n}$ the unique real subfield of $\mathbb{Q}\left(\mu_{q p^{n}}\right)$ which is the cyclic extension of the rational number field $\mathbb{Q}$ with degree $p^{n}$. Note that the Galois group of $\mathbb{B}_{\infty}=\bigcup_{n>0} \mathbb{B}_{n}$ over $\mathbb{Q}$ is isomorphic to the $p$-adic integer ring $\mathbb{Z}_{p}$ as additive group. The fields $\mathbb{B}_{\infty}$ and $\mathbb{B}_{n}$ are called the cyclotomic $\mathbb{Z}_{p}$-extension of $\mathbb{Q}$ and its $n$-th layer, respectively. We denote by $h_{n}$ the class number of $\mathbb{B}_{n}$. We consider the following problem.

Weber's class number problem. Is the class number $h_{n}$ equal to 1 for every non-negative integer $n$ ?

Manuscrit reçu le 6 novembre 2014, révisé le 27 juin 2015, accepté le 7 juillet 2015 .

Mathematics Subject Classification. 11R06, 11R18, 11R29.

Mots-clefs. Class number, $\mathbb{Z}_{p}$-extension, Height of algebraic number. 
In the case $p=2, \mathrm{H}$. Weber proved $h_{1}=h_{2}=h_{3}=1$. Later, several authors showed $h_{n}=1$ for $(p, n)=(2,4),(2,5),(3,1),(3,2),(3,3),(5,1)$ and $(7,1)$ (see [1], [3], [18] and [19]). And recently, J. C. Miller obtained striking results determining $h_{n}=1$ for $(p, n)=(2,6),(5,2),(11,1),(13,1)$, $(17,1)$ and $(19,1)$ (see [20] and [21]). However, calculating one class number by one gives information on the class numbers for only finitely many layers. Thus, we are lead to a problem of different aspect.

Problem 1.1. Fix a prime number $\ell$. Is the class number $h_{n}$ indivisible by $\ell$ for every non-negative integer $n$ ?

In the case $\ell=p, \mathrm{H}$. Weber [28] and K. Iwasawa [17] showed that $h_{n}$ is indivisible by $p$ for every non-negative integer $n$.

In the case $\ell \neq p$, by studying generalized Bernoulli numbers, L. C. Washington [27] proved that the quotient $h_{n} / h_{n-1}$ is indivisible by $\ell$ for sufficiently large $n$.

However, this result does not immediately imply the $\ell$-indivisibility of $h_{n}$. On the indivisibility problem, Washington [26] also showed that the minus part of the class number of $\mathbb{Q}\left(\mu_{5^{n+1}}\right)$ is indivisible by every prime number $\ell$ with $\ell^{8} \not \equiv 1(\bmod 100)$ for every non-negative integer $n$. Later, K. Horie $[7,8,9,10]$ and K. Horie-M. Horie $[11,12,13,14,15]$ made a breakthrough. Indeed, they succeeded in controlling cyclotomic units which relate to our class numbers.

We introduce notation before presenting a summary of their results. Let $f_{p}(\ell)$ be the order of $\ell$ modulo $q$ and $p^{s_{p}(\ell)}$ the exact power of $p$ dividing $\ell^{f_{p}(\ell)}-1$. And we define the set $D(p, s, f)$ of prime numbers to be

$$
D(p, s, f)=\left\{\ell \neq p \mid f_{p}(\ell)=f, s_{p}(\ell)=s\right\} .
$$

If $f$ divides $\varphi(q)$, where $\varphi$ is the Euler function, and $(p, s, f)$ is not in $\{(2,1,1),(2,1,2),(2,2,2)\}$, then $D(p, s, f)$ contains infinitely many prime numbers. Moreover, $D(p, s, f)$ can be written as a union of congruence classes of prime numbers modulo $p$-power. For example, $D(2,6,2)=\{\ell \equiv$ $31 \bmod 64\}$ and $D(3,1,2)=\{\ell \equiv 2 \bmod 9\} \cup\{\ell \equiv 5 \bmod 9\}$.

Theorem 1.2 (K. Horie-M. Horie). Let $p$ be a prime number.

(1) Let $s$ be a positive integer and $f$ a positive divisor of $\varphi(q)$. There exists an explicit positive constant $H(p, s, f)$ such that the class number $h_{n}$ is indivisible by every prime number $\ell$ in $D(p, s, f)$ with $\ell>H(p, s, f)$ for every non-negative integer $n$.

(2) If $p=2$, then the class number $h_{n}$ is indivisible by every prime number $\ell$ such that $\ell \not \equiv \pm 1(\bmod 8)$ for every non-negative integer $n$.

(3) If $3 \leq p \leq 23$, then the class number $h_{n}$, for every non-negative integer $n$, is indivisible by every prime number $\ell$ such that $\ell$ is a primitive root modulo $p^{2}$. 
Remark 1.3. They wrote $H(p, s, f)$ explicitly. We give a few of its numerical values at the end of this section.

T. Fukuda-K. Komatsu [5] proved the following theorem on the basis of the works of K. Horie.

Theorem 1.4 (T. Fukuda-K. Komatsu, [5]). Let $p=2$ and $\ell$ a prime number. Assume that $\ell<10^{9}$ or $\ell \neq \equiv \pm 1(\bmod 32)$. Then the class number $h_{n}$ is indivisible by $\ell$ for every non-negative integer $n$.

In our previous papers $[22,24,25]$, we proposed new methods for controlling cyclotomic units, which enabled us to prove the following theorem.

Theorem 1.5. Let $p, f$ and $s$ be the same as in Theorem 1.2 and $c=$ $(p-1) p^{s-1}$. We put

$$
G_{1}(p, s, f)= \begin{cases}(c !)^{1 / f} & \text { if } p=2, \\ \left(2^{c / 2} \cdot c !\right)^{1 / f} & \text { if } p=3, \\ \left(\left(\frac{\sqrt{6} p}{2}\right)^{c} \cdot c !\right)^{1 / f} & \text { if } p>3 .\end{cases}
$$

Assume that $\ell$ is greater than $G_{1}(p, s, f)$. Then the class number $h_{n}$ is indivisible by $\ell$ for every non-negative integer $n$.

The technical condition of Theorem 1.5 on the magnitude of $\ell$ is weaker than that of Theorem 1.2.

In this paper, we show the $\ell$-indivisibility under even weaker technical condition on the magnitude.

Theorem A. Let $p, f, s$ and $c$ be the same as in Theorem 1.5. We put

$$
\begin{aligned}
& G(p, s, f)=
\end{aligned}
$$




Then the class number $h_{n}$ is indivisible by every prime number $\ell$ in $D(p, s, f)$ with $\ell>G(p, s, f)$ for every non-negative integer $n$.

For example, $G(2,6,1)$ and $G(2,6,2)$ are smaller than $7.8 \times 10^{12}$ and $2.8 \times 10^{6}$, respectively.

Recalling Theorem 1.4, we see Theorem A implies the following corollary.

Corollary B. Let $p=2$. If $\ell$ is not congruent to \pm 1 modulo 64 , then $h_{n}$ is indivisible by $\ell$ for every non-negative integer $n$.

Example 1.6. We can compute $H, G_{1}$ and $G$. In the following table, we give a few examples of their values rounded up to two significant figures.

\begin{tabular}{|c|c|c|c|c|}
\hline$(p, s, f)$ & $D(p, s, f)$ & $H(p, s, f)$ & $G_{1}(p, s, f)$ & $G(p, s, f)$ \\
\hline$(2,5,1)$ & $\ell \equiv 33(\bmod 64)$ & $6.2 \times 10^{66}$ & $2.1 \times 10^{13}$ & $7.6 \times 10^{4}$ \\
\hline$(3,3,2)$ & $\ell \equiv 26,53(\bmod 81)$ & $5.5 \times 10^{32}$ & $1.9 \times 10^{9}$ & $4.3 \times 10^{4}$ \\
\hline$(5,1,1)$ & $\ell \equiv 6,11,16,21(\bmod 25)$ & $2.0 \times 10^{13}$ & $3.4 \times 10^{4}$ & $3.8 \times 10^{2}$ \\
\hline
\end{tabular}

Remark 1.7. In this paper, we don't study small primes. For small primes $\ell$, the reader should consult papers of H. Ichimura-S. Nakajima [16] or K. Horie-M. Horie [15]. In the case $\ell=2, H$. Ichimura-S. Nakajima showed that if $p \leq 509$, then $h_{n}$ is odd for every non-negative integer $n$. For small odd primes, there are several results proven by K. Horie-M. Horie. For example, they showed that if $3 \leq \ell \leq 13$ and $p \leq 101$, then $h_{n}$ is indivisible by $\ell$ for every non-negative integer $n$.

\section{Lemmas}

2.1. Horie unit. Let $p$ be a prime number. We put $\zeta_{n}=\exp \left(2 \pi \sqrt{-1} / p^{n}\right)$, $\mathbb{Q}\left(\mu_{p^{\infty}}\right)=\bigcup_{n=1}^{\infty} \mathbb{Q}\left(\mu_{p^{n}}\right), \sigma$ the topological generator of the Galois group $\operatorname{Gal}\left(\mathbb{Q}\left(\mu_{p^{\infty}}\right) / \mathbb{Q}\left(\mu_{q}\right)\right)$ with $\zeta_{n+2}^{\sigma}=\zeta_{n+2}^{5}$ if $p=2$ or $\zeta_{n+1}^{\sigma}=\zeta_{n+1}^{1+p}$ if $p>2$. Set $\tau_{n}=\sigma^{p^{n-1}}$. Then the restriction of $\sigma$ and $\tau_{n}$ to $\mathbb{B}_{n}$ generate the Galois groups $\operatorname{Gal}\left(\mathbb{B}_{n} / \mathbb{Q}\right)$ and $\operatorname{Gal}\left(\mathbb{B}_{n} / \mathbb{B}_{n-1}\right)$, respectively. Thus, we use the same symbols $\sigma$ and $\tau_{n}$ for their restriction to $\mathbb{B}_{n}$.

Let $E_{n}$ and $C_{n}$ be the group of units and of cyclotomic units of $\mathbb{B}_{n}$, respectively. Since

$$
\left[E_{n}^{1-\tau_{n}}: C_{n}^{1-\tau_{n}}\right]=h_{n} / h_{n-1},
$$

we study $E_{n}^{1-\tau_{n}}$ and $C_{n}^{1-\tau_{n}}$ (see [8]).

Since $\left(1-\tau_{n}\right)\left(1+\tau_{n}+\cdots+\tau_{n}^{p-1}\right)=0$, the ring $\mathbb{Z}\left[\zeta_{n}\right]$ acts on $\left(\mathbb{B}_{n}^{\times}\right)^{1-\tau_{n}}$, $E_{n}^{1-\tau_{n}}$ and $C_{n}^{1-\tau_{n}}$ via the isomorphism:

$$
\begin{aligned}
\mathbb{Z}\left[\operatorname{Gal}\left(\mathbb{B}_{n} / \mathbb{Q}\right)\right] /\left(1+\tau_{n}+\cdots+\tau_{n}^{p-1}\right) & \cong \mathbb{Z}\left[\zeta_{n}\right], \\
\sigma \bmod \left(1+\tau_{n}+\cdots+\tau_{n}^{p-1}\right) & \longmapsto \zeta_{n} .
\end{aligned}
$$

Hence we regard $\left(\mathbb{B}_{n}^{\times}\right)^{1-\tau_{n}}, E_{n}^{1-\tau_{n}}$ and $C_{n}^{1-\tau_{n}}$ as $\mathbb{Z}\left[\zeta_{n}\right]$-modules. 
We define the $n$-th Horie unit $\eta_{n}$ by

$$
\eta_{n}= \begin{cases}\frac{\zeta_{n+3}-\zeta_{n+3}^{-1}}{\sqrt{-1}\left(\zeta_{n+3}+\zeta_{n+3}^{-1}\right)} & \text { if } p=2, \\ N r_{\mathbb{Q}\left(\zeta_{n+1}+\zeta_{n+1}^{-1}\right) / \mathbb{B}_{n}}\left(\frac{\zeta_{n+1}-\zeta_{n+1}^{-1}}{\zeta_{1} \zeta_{n+1}-\zeta_{1}^{-1} \zeta_{n+1}^{-1}}\right) & \text { if } p>2 .\end{cases}
$$

In the case $p=2, \eta_{n}$ is also called Weber's normal unit (see e.g. [4] and [29]). The $n$-th Horie unit is essential for controlling our unit groups since $\eta_{n}^{1-\sigma}$ generates $C_{n}^{1-\tau_{n}}$ as $\mathbb{Z}\left[\zeta_{n}\right]$-module. Indeed, K. Horie showed the following lemma.

Lemma 2.1 (K. Horie, [8]). Let $\ell$ be a prime number different from $p$ and $F$ an intermediate field of $\mathbb{Q}\left(\zeta_{n}\right)$ and the decomposition field of $\ell$ for $\mathbb{Q}\left(\zeta_{n}\right) / \mathbb{Q}$. Then $\ell$ divides the integer $h_{n} / h_{n-1}$ if and only if there exists a prime ideal $\mathfrak{L}$ of $F$ dividing $\ell$ such that $\eta_{n}^{\alpha}$ is an $\ell$-th power in $E_{n}$ for every element $\alpha$ of the integral ideal $\ell \mathfrak{L}^{-1}$ of $F$.

In the case where $p$ is an odd prime number, we consider another cyclotomic unit $\delta_{n}$ defined by

$$
\delta_{n}=N r_{\mathbb{Q}\left(\zeta_{n+1}+\zeta_{n+1}^{-1}\right) / \mathbb{B}_{n}}\left(\frac{\left(\zeta_{n+1}-\zeta_{n+1}^{-1}\right)^{p}}{\zeta_{n}-\zeta_{n}^{-1}}\right)
$$

which enables us to obtain precise information on the Horie unit $\eta_{n}$ through the relation

$$
\delta_{n}^{1-\tau_{n}}=\eta_{n}^{p}
$$

For simplicity, we also put $\delta_{n}=\eta_{n}$ if $p=2$.

2.2. Height of unit. Let $\varepsilon$ be a totally real unit of degree $N$ with conjugates $\varepsilon_{1}=\varepsilon, \varepsilon_{2}, \cdots, \varepsilon_{N}$. We define the height of unit $\varepsilon$.

Definition 2.2 (Height of unit). We define the $L_{2}$-height of the Dirichlet embedding of $\varepsilon$ by

$$
h t(\varepsilon)=\sqrt{\sum_{i=1}^{N}\left(\log \left|\varepsilon_{i}\right|\right)^{2}}
$$

For simplicity, we call $h t(\varepsilon)$ the height of $\varepsilon$.

The height of totally real units allows quantitative control as described below.

Lemma 2.3. Let $\varepsilon$ be a totally real unit of degree $N>1$. We put $C=$ $\left|N r_{\mathbb{Q}(\varepsilon) / \mathbb{Q}}\left(\varepsilon^{2}-1\right)\right|$. Then we have

$$
h t(\varepsilon) \geq \sqrt{N} \log \left(\frac{C^{1 / N}+\sqrt{C^{2 / N}+4}}{2}\right) .
$$


In particular, we have

$$
h t(\varepsilon) \geq \sqrt{N} \log \left(\frac{1+\sqrt{5}}{2}\right) .
$$

Proof. Let

$$
M(\varepsilon)=\prod_{i=1}^{N} \max \left\{1,\left|\varepsilon_{i}\right|\right\}
$$

be the Mahler measure of $\varepsilon$. Then we have

$$
M(\varepsilon) \geq\left(\frac{C^{1 / N}+\sqrt{C^{2 / N}+4}}{2}\right)^{N / 2}
$$

(see e.g. [24, Theorem 2.2]).

On the other hand, we know that

$$
\log M(\varepsilon)=\frac{1}{2} \sum_{i=1}^{N}|\log | \varepsilon_{i}||
$$

Hence we obtain

$$
\frac{\sqrt{N}}{2} h t(\varepsilon) \geq \log M(\varepsilon)
$$

from the Cauchy-Schwarz inequality. The assertions follow after (2.3) and (2.4).

Up to this point, $\varepsilon$ is just an irrational totally real unit. To apply Lemma 2.3 to our case, we have the following lemma.

Lemma 2.4. Let $\varepsilon$ be a unit in $E_{n} \backslash E_{n-1}$ with $N r_{\mathbb{B}_{n} / \mathbb{B}_{n-1}}(\varepsilon)=1$.

(1) ([23, Lemma 5.2]) If $p=2$, then $\varepsilon$ is congruent to 1 modulo 2 . In particular, we have

$$
\left|N r_{\mathbb{B}_{n} / \mathbb{Q}}\left(\varepsilon^{2}-1\right)\right| \geq 4^{2^{n}}
$$

(2) ([24, Lemma 9.1]) If $p$ is odd, then we know

$$
\left|N r_{\mathbb{B}_{n} / \mathbb{Q}}\left(\varepsilon^{2}-1\right)\right| \geq p^{\left(p^{n}-1\right) /(p-1)} .
$$

Proof. For the convenience of the reader, we give an alternative proof of (1).

Let $\varepsilon$ be a unit in $E_{n} \backslash E_{n-1}$ with $N r_{\mathbb{B}_{n} / \mathbb{B}_{n-1}}(\varepsilon)=1$. Then there exist integers $b_{0}, \cdots, b_{N-1}$ such that

$$
\varepsilon=b_{0}+\sum_{i=1}^{N-1} b_{i}\left(\zeta_{n+2}^{i}+\zeta_{n+2}^{-i}\right) .
$$

We put

$$
\begin{aligned}
& \gamma_{+}(\varepsilon)=b_{0}+b_{2}\left(\zeta_{n+2}^{2}+\zeta_{n+2}^{-2}\right)+\cdots+b_{N-2}\left(\zeta_{n+2}^{N-2}+\zeta_{n+2}^{-N+2}\right), \\
& \gamma_{-}(\varepsilon)=b_{1}\left(\zeta_{n+2}+\zeta_{n+2}^{-1}\right)+\cdots+b_{N-1}\left(\zeta_{n+2}^{N-1}+\zeta_{n+2}^{-N+1}\right) .
\end{aligned}
$$


Then we have

$$
\varepsilon=\gamma_{+}(\varepsilon)+\gamma_{-}(\varepsilon), \quad \gamma_{+}(\varepsilon)^{\tau_{n}}=\gamma_{+}(\varepsilon), \quad \gamma_{-}(\varepsilon)^{\tau_{n}}=-\gamma_{-}(\varepsilon)
$$

Since $N r_{\mathbb{B}_{n} / \mathbb{B}_{n-1}}(\varepsilon)=1$, we obtain

$$
\gamma_{+}(\varepsilon)^{2}-\gamma_{-}(\varepsilon)^{2}=1
$$

This implies

$$
\left(\gamma_{+}(\varepsilon)-1\right)\left(\gamma_{+}(\varepsilon)+1\right)=\gamma_{-}(\varepsilon)^{2} .
$$

Let $\mathfrak{p}_{n}$ be the prime ideal of $\mathbb{B}_{n}$ lying above 2 . We denote by $\nu_{2}$ the additive $\mathfrak{p}_{n}$-adic valuation nomalized by $\nu_{2}\left(\zeta_{n+2}+\zeta_{n+2}^{-1}\right)=1$. Assume that $\nu_{2}\left(\gamma_{+}(\varepsilon)-1\right)<N$. Then we have

$$
\nu_{2}\left(\gamma_{+}(\varepsilon)-1\right)=\nu_{2}\left(\gamma_{+}(\varepsilon)+1\right)=\nu_{2}\left(\gamma_{-}(\varepsilon)\right)
$$

from $(2.5)$. However, $\nu_{2}\left(\gamma_{+}(\varepsilon)-1\right)$ and $\nu_{2}\left(\gamma_{+}(\varepsilon)+1\right)$ are even and $\nu_{2}\left(\gamma_{-}(\varepsilon)\right)$ is odd or $\infty$. This is a contradiction.

Thus, we have $\nu_{2}\left(\gamma_{+}(\varepsilon)-1\right) \geq N$. Therefore, we obtain $\gamma_{+}(\varepsilon) \equiv 1$ $(\bmod 2)$ and $\gamma_{-}(\varepsilon) \equiv 0(\bmod 2)$, that is, $\varepsilon \equiv 1(\bmod 2)$.

By combining Lemma 2.3 and Lemma 2.4, we obtain the following lemma.

Lemma 2.5. Let $\varepsilon$ be a unit in $E_{n} \backslash E_{n-1}$ with $N r_{\mathbb{B}_{n} / \mathbb{B}_{n-1}}(\varepsilon)=1$.

(1) If $p=2$, then we have

$$
h t(\varepsilon) \geq \sqrt{2^{n}} \log (2+\sqrt{5}) .
$$

(2) If $p$ is an odd prime number, then we have

$$
h t(\varepsilon) \geq \sqrt{p^{n}} \log \left(\frac{p^{\left(p^{n}-1\right) / p^{n}(p-1)}+\sqrt{p^{2\left(p^{n}-1\right) / p^{n}(p-1)}+4}}{2}\right) .
$$

2.3. Geometry of numbers. For applying Lemma 2.1, it is desirable to choose an $\alpha$ in an ideal of $F$ so that $h t\left(\delta_{n}^{\alpha}\right)$ is small.

Let $r$ and $n$ be positive integers with $r \leq n$ and $p^{r} \neq 2$. We put $d=\varphi\left(p^{r}\right)$ where $\varphi$ is the Euler function. We start with geometry of numbers for an arbitrary ideal $\mathfrak{a}$ of $\mathbb{Z}\left[\zeta_{r}\right]\left(F=\mathbb{Q}\left(\zeta_{r}\right)\right)$.

Definition 2.6. We define a map $\lambda_{n}$ from $E_{n}$ to $\mathbb{R}^{p^{n}}$ by

$$
\lambda_{n}(\varepsilon)=\left(\log |\varepsilon|, \log \left|\varepsilon^{\sigma}\right|, \cdots, \log \left|\varepsilon^{\sigma^{p^{n}-1}}\right|\right) .
$$

We define $d$-dimensional $\mathbb{R}$-vector space

$$
V_{n}=\mathbb{R} \lambda_{n}\left(\delta_{n}\right) \oplus \mathbb{R} \lambda_{n}\left(\delta_{n}^{\zeta_{r}}\right) \oplus \cdots \oplus \mathbb{R} \lambda_{n}\left(\delta_{n}^{\zeta_{r}^{d-1}}\right)
$$

with standard metric. Let $\mathfrak{a}$ be an integral ideal of $\mathbb{Q}\left(\zeta_{r}\right)$. We associate the lattice

$$
\Lambda=\lambda_{n}(\mathfrak{a})=\left\{\lambda_{n}\left(\delta_{n}^{\alpha}\right) ; \alpha \in \mathfrak{a}\right\}
$$


in $V_{n}$ with $\mathfrak{a}$.

Let $\beta_{1}, \cdots, \beta_{d}$ be a $\mathbb{Z}$-basis of $\mathfrak{a}$. The metric of $V_{n}$ induces the quadratic form

$$
F_{\mathfrak{a}}\left(x_{1}, \cdots, x_{d}\right)=h t\left(\delta_{n}^{\sum_{i=1}^{d} x_{i} \beta_{i}}\right)^{2} .
$$

Then

$$
F_{\mathfrak{a}}\left(x_{1}, \cdots, x_{d}\right)=\sum_{j=0}^{p^{n}-1}\left(\sum_{i=1}^{d} x_{i} \log \left|\delta_{n}^{\beta_{i} \sigma^{j}}\right|\right)^{2}
$$

is a positive definite quadratic form in $d$ variables and of determinant $\operatorname{vol}^{(d)}(\Lambda)^{2}$ where $\operatorname{vol}^{(d)}$ is the $d$-dimensional volume on $V_{n}$.

We will detect a non-zero lattice point of $\Lambda$ by the following theorem in geometry of numbers.

Theorem 2.7 (Blichfeldt, [2, Theorem II]). Let $\Lambda$ be a lattice in the metric vector space of dimension d. Then there exists a non-zero vector $v$ of $\Lambda$ such that

$$
|v|^{2} \leq \frac{2}{\pi}\left(\Gamma\left(1+\frac{d+2}{2}\right)\right)^{2 / d} \operatorname{vol}^{(d)}(\Lambda)^{2 / d} .
$$

In our setting of $\Lambda$, this implies the following lemma.

Lemma 2.8. Let $\mathfrak{a}$ be an integral ideal of $\mathbb{Z}\left[\zeta_{r}\right]$. Then there exists a nonzero element $\alpha$ in $\mathfrak{a}$ such that

$$
h t\left(\delta_{n}^{\alpha}\right) \leq \sqrt{\frac{2}{\pi}}\left(\frac{d+2}{2} !\left[\mathbb{Z}\left(\zeta_{r}\right): \mathfrak{a}\right] \operatorname{vol}^{(d)}\left(\lambda_{n}\left(\mathbb{Z}\left[\zeta_{r}\right]\right)\right)\right)^{1 / d} .
$$

\section{Proof in the case $p=2$}

In this section, we prove Theorem $\mathrm{A}$ for $p=2$.

Let $p=2, \ell$ a prime number in $D(2, s, f)$ and $n$ a positive integer. We put $r=\min \{n, s\}$ and $d=2^{r-1}$.

\subsection{Height of Horie unit for $p=2$.}

Lemma 3.1. Assume $p=2$. We have

$$
h t\left(\eta_{n}\right) \leq \frac{\pi}{2} \sqrt{2^{n}} .
$$

Proof. We rewrite (2.1) as

$$
\eta_{n}=\frac{\zeta_{n+3}-\zeta_{n+3}^{-1}}{\zeta_{n+3}^{1+2^{n+1}}-\zeta_{n+3}^{-1-2^{n+1}}}
$$

Then, we see

$$
\eta_{n}^{\sigma^{i}}=\tan \frac{5^{i} \pi}{2^{n+2}}
$$


Hence we obtain

$$
\begin{aligned}
h t\left(\eta_{n}\right)^{2} & =\sum_{i=1}^{2^{n}}\left(\log \left|\tan \frac{(2 i-1) \pi}{2^{n+2}}\right|\right)^{2} \\
& =2 \sum_{i=1}^{2^{n-1}}\left(\log \tan \frac{(2 i-1) \pi}{2^{n+2}}\right)^{2}
\end{aligned}
$$

Since

$$
\frac{d}{d \theta}(\log \tan \theta)^{2}=\frac{2 \log \tan \theta}{\sin \theta \cos \theta}<0
$$

and

$$
\frac{d^{2}}{d \theta^{2}}(\log \tan \theta)^{2}=\frac{8(1-\cos 2 \theta \log \tan \theta)}{(\sin 2 \theta)^{2}}>0
$$

for $0<\theta<\pi / 4$, we have

$$
\begin{aligned}
2 \sum_{i=1}^{2^{n-1}}\left(\log \left|\tan \frac{(2 i-1) \pi}{2^{n+2}}\right|\right)^{2} & \leq \frac{2^{n+2}}{\pi} \int_{0}^{\pi / 4}(\log \tan \theta)^{2} d \theta \\
& =\frac{\pi^{2}}{4} 2^{n}
\end{aligned}
$$

3.2. Volume of lattice for $\boldsymbol{p}=\mathbf{2}$. We assume $n \geq 2$. Then we have the following lemma.

Lemma 3.2. Assume $p=2$. Let $\mathfrak{L}$ be a prime ideal of $\mathbb{Q}\left(\zeta_{r}\right)$ lying above $\ell$. We have

$$
\operatorname{vol}^{(d)}\left(\lambda_{n}\left(\left(1-\zeta_{r}\right) \ell \mathfrak{L}^{-1}\right)\right) \leq 2 \ell^{d-f}\left(\frac{\pi}{2} \sqrt{2^{n}}\right)^{d} .
$$

Proof. Note that

$$
\operatorname{vol}^{(d)}\left(\lambda_{n}\left(\left(1-\zeta_{r}\right) \ell \mathfrak{L}^{-1}\right)\right)=\left[\mathbb{Z}\left[\zeta_{r}\right]:\left(1-\zeta_{r}\right) \ell \mathfrak{L}^{-1}\right] \operatorname{vol}^{(d)}\left(\lambda_{n}\left(\mathbb{Z}\left[\zeta_{r}\right]\right)\right) .
$$

From $r \leq s$, we have

$$
\left[\mathbb{Z}\left[\zeta_{r}\right]:\left(1-\zeta_{r}\right) \ell \mathfrak{L}^{-1}\right]=2 \ell^{d-f} .
$$

Since $h t\left(\eta_{n}\right)=h t\left(\eta_{n}^{\zeta_{r}}\right)=\cdots=h t\left(\eta_{n}^{\zeta_{r}^{d-1}}\right)$, we obtain

$$
\begin{aligned}
\operatorname{vol}^{(d)}\left(\lambda_{n}\left(\mathbb{Z}\left[\zeta_{r}\right]\right)\right) & \leq h t\left(\eta_{n}\right)^{d} \\
& \leq\left(\frac{\pi}{2} \sqrt{2^{n}}\right)^{d} .
\end{aligned}
$$


3.3. Concluding the proof of Theorem $\mathrm{A}$ for $\boldsymbol{p}=\mathbf{2}$. We prove the contrapositive. Suppose that $\ell$ divides $h_{n} / h_{n-1}$. It is sufficient to show that $\ell \leq G(2, s, f)$.

Since $h_{1}=1$, we may assume that $n \geq 2$. From Lemma 2.1, there exists a prime ideal $\mathfrak{L}$ in $\mathbb{Q}\left(\zeta_{r}\right)$ lying above $\ell$ such that $\eta_{n}^{\alpha}$ is an $\ell$-th power in $E_{n}$ for every element $\alpha$ of $\ell \mathfrak{L}^{-1}$.

We put $\mathfrak{a}=\left(1-\zeta_{r}\right) \ell \mathfrak{L}^{-1}$. From Lemmas 2.8 and 3.2 , there exists a non-zero element $\alpha$ in $\ell \mathfrak{L}^{-1}$ such that

$$
h t\left(\eta_{n}^{\left(1-\zeta_{r}\right) \alpha}\right) \leq \sqrt{\frac{2}{\pi}}\left(\frac{d+2}{2} ! 2 \ell^{d-f}\left(\frac{\pi}{2} \sqrt{2^{n}}\right)^{d}\right)^{1 / d} .
$$

From Lemma 2.1, there exists a unit $\varepsilon$ in $E_{n}$ such that $\eta_{n}^{\alpha}=\varepsilon^{\ell}$. Therefore, we have

$$
\eta_{n}^{\left(1-\zeta_{r}\right) \alpha}=\left(\varepsilon^{1-\zeta_{r}}\right)^{\ell} .
$$

Since $N r_{\mathbb{B}_{n} / \mathbb{B}_{n-1}}\left(\eta_{n}\right)=-1$ and $\left(1-\zeta_{r}\right) \alpha$ is non-zero, the degree of $\varepsilon^{1-\zeta_{r}}$ is $2^{n}$ and $N r_{\mathbb{B}_{n} / \mathbb{B}_{n-1}}\left(\varepsilon^{1-\zeta_{r}}\right)=1$. Hence we have

$$
h t\left(\varepsilon^{1-\zeta_{r}}\right) \geq \sqrt{2^{n}} \log (2+\sqrt{5}) .
$$

from Lemma 2.5 (1).

From (3.3), (3.4) and (3.5), we obtain

$$
\ell \sqrt{2^{n}} \log (2+\sqrt{5}) \leq \sqrt{\frac{2}{\pi}}\left(\frac{d+2}{2} ! 2 \ell^{d-f}\left(\frac{\pi}{2} \sqrt{2^{n}}\right)^{d}\right)^{1 / d} .
$$

This implies

$$
\ell \leq\left(2\left(\frac{\sqrt{\pi}}{\sqrt{2} \log (2+\sqrt{5})}\right)^{d} \frac{d+2}{2} !\right)^{1 / f} .
$$

Since $c=2^{s-1}$ and $s \geq r$, we have $c \geq d$. Hence we can replace $d$ with $c$. Therefore, we have

$$
\ell \leq\left(2\left(\frac{\sqrt{\pi}}{\sqrt{2} \log (2+\sqrt{5})}\right)^{c} \frac{c+2}{2} !\right)^{1 / f}=G(2, s, f) .
$$

\section{Proof in the case $p \geq 3$}

In this section, we prove Theorem $\mathrm{A}$ for $p \geq 3$.

Let $p$ be a prime number with $p \geq 3, \ell$ a prime number in $D(p, s, f)$ and $n$ a positive integer. We put $r=\min \{n, s\}$ and $d=(p-1) p^{r-1}$. 
4.1. Height of $\boldsymbol{\delta}_{\boldsymbol{n}}$. From the definition of $\delta_{n}$ and the Cauchy-Schwarz inequality, we obtain

$$
\begin{aligned}
h t\left(\delta_{n}\right)^{2} & =\sum_{i=0}^{p^{n}-1}\left(\log \left|\delta_{n}^{\sigma^{i}}\right|\right)^{2} \\
& \leq \frac{p-1}{4} \sum_{i=1, p \nmid i}^{p^{n+1}-1}\left(\log \left|\frac{\left(\zeta_{n+1}^{i}-\zeta_{n+1}^{-i}\right)^{p}}{\zeta_{n}^{i}-\zeta_{n}^{-i}}\right|\right)^{2} \\
& =\frac{p-1}{4} \sum_{i=1, p \nmid i}^{p^{n+1}-1}\left(p \log \left|2 \sin \left(\frac{2 i \pi}{p^{n+1}}\right)\right|-\log \left|2 \sin \left(\frac{2 i \pi}{p^{n}}\right)\right|\right)^{2} .
\end{aligned}
$$

Since 2 acts on $\left(\mathbb{Z} / p^{n+1} \mathbb{Z}\right)^{\times}$, we have

$$
\begin{aligned}
\frac{p-1}{4} & \sum_{i=1, p \nmid i}^{p^{n+1}-1}\left(p \log \left|2 \sin \left(\frac{2 i \pi}{p^{n+1}}\right)\right|-\log \left|2 \sin \left(\frac{2 i \pi}{p^{n}}\right)\right|\right)^{2} \\
= & \frac{p-1}{4} \sum_{i=1, p \nmid i}^{p^{n+1}-1}\left(p \log \left|2 \sin \left(\frac{i \pi}{p^{n+1}}\right)\right|-\log \left|2 \sin \left(\frac{i \pi}{p^{n}}\right)\right|\right)^{2} \\
= & \frac{p-1}{4} \sum_{i=1, p \nmid i}^{p^{n}-1} \sum_{j=0}^{p-1}\left(p \log \left|2 \sin \left(\frac{i \pi}{p^{n+1}}+\frac{j \pi}{p}\right)\right|-\log \left|2 \sin \left(\frac{i \pi}{p^{n}}\right)\right|\right)^{2} \\
= & \frac{p(p-1)}{4} \sum_{i=1, p \nmid i}^{p^{n}-1} g\left(\frac{i \pi}{p^{n+1}}\right) \\
= & \frac{p(p-1)}{4} \sum_{k=0}^{p^{n-1}-1} \sum_{i=1}^{p-1} g\left(\frac{(i+p k) \pi}{p^{n+1}}\right)
\end{aligned}
$$

where

$$
g(\theta)=\frac{1}{p} \sum_{j=0}^{p-1}\left(p \log \left|2 \sin \left(\theta+\frac{j \pi}{p}\right)\right|-\log |2 \sin (p \theta)|\right)^{2} .
$$

From the equality

$$
\prod_{j=0}^{p-1}\left(\zeta_{1}^{j} x-\zeta_{1}^{-j} x^{-1}\right)=x^{p}-x^{-p}
$$

we get

$$
\prod_{j=0}^{p-1}\left|2 \sin \left(\theta+\frac{j \pi}{p}\right)\right|=|2 \sin (p \theta)|
$$


Hence we obtain

$$
g(\theta)=p \sum_{j=0}^{p-1}\left(\log \left|2 \sin \left(\theta+\frac{j \pi}{p}\right)\right|\right)^{2}-(\log |2 \sin (p \theta)|)^{2} .
$$

In order to get an upper bound on the height of $\delta_{n}$, we give another description of $g(\theta)$. We put $C(\theta, \alpha)=(\log |2 \sin \theta|-\log |2 \sin (\theta+\alpha)|)^{2}$. Then we get

$$
\begin{aligned}
\frac{1}{2} \sum_{i^{\prime}=0}^{p-1} \sum_{j^{\prime}=0}^{p-1} C\left(\theta+\frac{i^{\prime} \pi}{p}, \frac{j^{\prime} \pi}{p}\right) \\
=\frac{1}{2} \sum_{i^{\prime}=0}^{p-1} \sum_{j^{\prime}=0}^{p-1}\left(\log \left|2 \sin \left(\theta+\frac{i^{\prime} \pi}{p}\right)\right|-\log \left|2 \sin \left(\theta+\frac{i^{\prime} \pi}{p}+\frac{j^{\prime} \pi}{p}\right)\right|\right)^{2} \\
=p \sum_{j=0}^{p-1}\left(\log \left|2 \sin \left(\theta+\frac{j \pi}{p}\right)\right|\right)^{2} \\
\quad-\sum_{i^{\prime}=0}^{p-1} \log \left|2 \sin \left(\theta+\frac{i \pi}{p}\right)\right| \sum_{j^{\prime}=0}^{p-1} \log \left|2 \sin \left(\theta+\frac{i^{\prime} \pi}{p}+\frac{j^{\prime} \pi}{p}\right)\right| \\
=p \sum_{j=0}^{p-1}\left(\log \left|2 \sin \left(\theta+\frac{j \pi}{p}\right)\right|\right)^{2}-(\log |2 \sin (p \theta)|)^{2} .
\end{aligned}
$$

This implies

$$
g(\theta)=\frac{1}{2} \sum_{i^{\prime}=0}^{p-1} \sum_{j^{\prime}=0}^{p-1} C\left(\theta+\frac{i^{\prime} \pi}{p}, \frac{j^{\prime} \pi}{p}\right)
$$

Note that

$$
\begin{aligned}
& \frac{1}{2}\left(\frac{d}{d \theta}\right)^{2} C(\theta, \alpha) \\
& \quad=\left(\frac{\cos \theta}{\sin \theta}-\frac{\cos (\theta+\alpha)}{\sin (\theta+\alpha)}\right)^{2}-\left(\frac{1}{(\sin \theta)^{2}}-\frac{1}{(\sin (\theta+\alpha))^{2}}\right) \log \left|\frac{\sin \theta}{\sin (\theta+\alpha)}\right| .
\end{aligned}
$$

We see

$$
\left(\frac{d}{d \theta}\right)^{2} C(\theta, \alpha) \geq 0
$$

for $\theta$ and $\theta+\alpha$ not equal to multiples of $\pi$. Therefore, $g(\theta)$ is a convex function on $] 0, \pi / p[$ from (4.2).

Now we need the following proposition. 
Proposition 4.1. Let $M$ be a positive integer and $F(\theta)$ a convex function on an interval $] a, b\left[\right.$. Assume that $\int_{a}^{b} F(\theta) d \theta$ is convergent. Then we have

$$
\sum_{i=1}^{M} F\left(a+\frac{b-a}{M+1} i\right) \leq \frac{M}{b-a} \int_{a}^{b} F(\theta) d \theta .
$$

Note that $g(\theta)$ is a convex function on the interval $] 0, \pi / p[$. By applying Proposition 4.1 for $M=p-1, a=k \pi / p^{n}$ and $b=(k+1) \pi / p^{n}$, we see

$$
\sum_{i=1}^{p-1} g\left(\frac{(i+p k) \pi}{p^{n+1}}\right) \leq \frac{(p-1) p^{n}}{\pi} \int_{k \pi / p^{n}}^{(k+1) \pi / p^{n}} g(\theta) d \theta .
$$

for $0 \leq k \leq p^{n-1}-1$. Therefore, by taking sum, we obtain

$$
h t\left(\delta_{n}\right)^{2} \leq \frac{(p-1)^{2} p^{n+1}}{4 \pi} \int_{0}^{\pi / p} g(\theta) d \theta .
$$

From the equality (4.1), we have

$$
\begin{array}{rl}
\int_{0}^{\pi / p} & g(\theta) d \theta \\
= & p \sum_{j=0}^{p-1} \int_{0}^{\pi / p}\left(\log \left|2 \sin \left(\theta+\frac{j \pi}{p}\right)\right|\right)^{2} d \theta-\int_{0}^{\pi / p}(\log |2 \sin (p \theta)|)^{2} d \theta \\
= & p \sum_{j=0}^{p-1} \int_{j \pi / p}^{(j+1) \pi / p}(\log |2 \sin \theta|)^{2} d \theta-\frac{1}{p} \int_{0}^{\pi}(\log |2 \sin \theta|)^{2} d \theta \\
= & \frac{p^{2}-1}{p} \int_{0}^{\pi}(\log |2 \sin \theta|)^{2} d \theta \\
= & \frac{\left(p^{2}-1\right) \pi^{3}}{12 p} .
\end{array}
$$

Hence we obtain

$$
h t\left(\delta_{n}\right)^{2} \leq \frac{p^{n}(p-1)^{3}(p+1) \pi^{2}}{48}
$$

Therefore, we get the following lemma.

Lemma 4.2. Assume $p \geq 3$. We have

$$
h t\left(\delta_{n}\right) \leq \frac{(p-1) \pi \sqrt{3\left(p^{2}-1\right)}}{12} \sqrt{p^{n}} .
$$

4.2. Volume of lattice for $\boldsymbol{p} \geq \mathbf{3}$. Let $m$ and $d$ be positive integers with $m \leq d$ and $V$ a $d$-dimensional $\mathbb{R}$-vector space. For $v_{0}, v_{1}, \cdots, v_{m}$ in $V$, we define the parallelotope $S\left(v_{0}, v_{1}, \cdots, v_{m}\right)$ by

$$
S\left(v_{0}, v_{1}, \cdots, v_{m}\right)=\left\{\sum_{i=0}^{m} t_{i} v_{i} ; 0 \leq t_{i} \leq 1, \sum_{i=0}^{m} t_{i}=1\right\} .
$$


We quote the following estimate ([6, Theorem 2.2]) of its volume.

Proposition 4.3. If $\left\|v_{0}\right\|=\left\|v_{1}\right\|=\cdots=\left\|v_{m}\right\|=h$, then we have

$$
\operatorname{vol}^{(m)}\left(S\left(v_{0}, v_{1}, \cdots, v_{m}\right)\right) \leq \frac{(m+1)^{(m+1) / 2}}{m ! m^{m / 2}} h^{m} .
$$

We put

$$
Q\left(v_{1}, \cdots, v_{m}\right)=\left\{\sum_{i=1}^{m} t_{i} v_{i} ; 0 \leq t_{i} \leq 1\right\}
$$

and

$Q_{j, k}=Q\left(\lambda_{n}\left(\delta_{n}^{\zeta_{r}^{j}}\right), \lambda_{n}\left(\delta_{n}^{\zeta_{n}^{j}} \zeta_{1}\right), \cdots, \lambda_{n}\left(\delta_{n}^{\zeta_{r}^{j} \zeta_{1}^{k-1}}\right), \lambda_{n}\left(\delta_{n}^{\zeta_{\zeta}^{j} \zeta_{1}^{k+1}}\right), \cdots, \lambda_{n}\left(\delta_{n}^{\zeta^{j} \zeta_{1}^{p-1}}\right)\right)$.

Then we have the following proposition.

Proposition 4.4. For $0 \leq j \leq p^{r-1}-1$, we have

$$
\begin{aligned}
\frac{p}{(p-1) !} \operatorname{vol}^{(p-1)}\left(Q_{j, p-1}\right) & \\
& =\operatorname{vol}^{(p-1)}\left(S\left(\lambda_{n}\left(\delta_{n}^{\zeta_{r}^{j}}\right), \lambda_{n}\left(\delta_{n}^{\zeta_{n}^{j} \zeta 1}\right), \cdots, \lambda_{n}\left(\delta_{n}^{\zeta_{n}^{j} \zeta_{1}^{p-1}}\right)\right)\right) .
\end{aligned}
$$

Proof. Note that, since $\sum_{k=0}^{p-1} \lambda_{n}\left(\delta_{n}^{\zeta_{n}^{i} \zeta_{1}^{k}}\right)=0$, we have

$$
\begin{aligned}
\operatorname{vol}^{(p-1)}\left(S\left(\lambda_{n}\left(\delta_{n}^{\zeta_{r}^{j}}\right), \lambda_{n}\left(\delta_{n}^{\zeta_{n}^{j} \zeta_{1}}\right), \cdots, \lambda_{n}\left(\delta_{n}^{\zeta_{r}^{j} \zeta_{1}^{p-1}}\right)\right)\right) & \\
& =\frac{1}{(p-1) !} \sum_{k=0}^{p-1} \operatorname{vol}^{(p-1)}\left(Q_{j, k}\right) .
\end{aligned}
$$

and $\operatorname{vol}^{(p-1)}\left(Q_{j, k}\right)=\operatorname{vol}^{(p-1)}\left(Q_{j, k^{\prime}}\right)$ for $0 \leq k, k^{\prime} \leq p-1$. Therefore, we obtain the assertion.

Then we obtain the following lemma.

Lemma 4.5. Assume $p \geq 3$. Let $\mathfrak{L}$ be a prime ideal of $\mathbb{Q}\left(\zeta_{r}\right)$ lying above $\ell$. Then we have

$$
\operatorname{vol}^{(d)}\left(\lambda_{n}\left(\left(1-\zeta_{1}\right) \ell \mathfrak{L}^{-1}\right)\right) \leq \ell^{d-f}\left(\frac{\pi(p-1) p^{p / 2(p-1)} \sqrt{p+1}}{4 \sqrt{3}} \sqrt{p^{n}}\right)^{d} .
$$


Proof. From Propositions 4.3 and 4.4, we have

$$
\begin{aligned}
{\left[\mathbb{Z}\left[\zeta_{r}\right]:\left(1-\zeta_{1}\right) \ell \mathfrak{L}^{-1}\right] \operatorname{vol}^{(d)}\left(\lambda_{n}\left(\mathbb{Z}\left[\zeta_{r}\right]\right)\right) } & \\
& =p^{p^{r-1}} \ell^{d-f} \operatorname{vol}^{(d)}\left(\lambda_{n}\left(\mathbb{Z}\left[\zeta_{r}\right]\right)\right) \\
& \leq p^{p^{r-1}} \ell^{d-f} \prod_{j=0}^{p^{r-1}-1} \operatorname{vol}^{(p-1)}\left(Q_{j, p-1}\right) \\
& \leq p^{p^{r-1}} \ell^{d-f} \prod_{j=0}^{p^{r-1}-1} \frac{(p-1) !}{p} \operatorname{vol}^{(p-1)} \\
& \times\left(S\left(\lambda_{n}\left(\delta_{n}^{\zeta_{r}^{j}}\right), \lambda_{n}\left(\delta_{n}^{\zeta_{r}^{j}} \zeta_{1}\right), \cdots, \lambda_{n}\left(\delta_{n}^{\zeta_{r}^{j} \zeta_{1}^{p-1}}\right)\right)\right) \\
& \leq \ell^{d-f} \frac{p^{p^{r} / 2}}{(p-1)^{d / 2}} h t\left(\delta_{n}\right)^{d} .
\end{aligned}
$$

From Lemma 4.2, we obtain the assertion.

4.3. Concluding the proof of Theorem A for odd $\boldsymbol{p}$. We prove the contrapositive. Suppose that $\ell$ divides $h_{n} / h_{n-1}$. It is sufficient to show that $\ell \leq G(p, s, f)$. Since $h_{n}=1$ for $(p, n)=(3,1),(3,2),(3,3),(5,1),(5,2)$, $(7,1),(11,1),(13,1),(17,1)$ and $(19,1)$, we may assume that $n \geq 4$ if $p=3$, $n \geq 3$ if $p=5$ and $n \geq 2$ if $7 \leq p \leq 17$.

From Lemma 2.1 , there exist a prime ideal $\mathfrak{L}$ in $\mathbb{Q}\left(\zeta_{r}\right)$ lying above $\ell$ such that $\eta_{n}^{\alpha}$ is an $\ell$-th power in $E_{n}$ for every element $\alpha$ of $\ell \mathfrak{L}^{-1}$.

We put $\mathfrak{a}=\left(1-\zeta_{1}\right) \ell \mathfrak{L}^{-1}$. From Lemmas 2.8 and 4.5 , there exists a non-zero element $\alpha$ in $\ell \mathfrak{L}^{-1}$ such that

$$
h t\left(\delta_{n}^{\left(1-\zeta_{1}\right) \alpha}\right) \leq \sqrt{\frac{2}{\pi}}\left(\frac{d+2}{2} ! \ell^{d-f}\left(\frac{\pi(p-1) p^{p / 2(p-1)} \sqrt{p+1}}{4 \sqrt{3}} \sqrt{p^{n}}\right)^{d}\right)^{1 / d} .
$$

From (2.2), we have $\delta_{n}^{1-\zeta_{1}}=\eta_{n}^{p}$. Moreover, from Lemma 2.1, there exist a unit $\varepsilon$ in $E_{n}$ such that $\eta_{n}^{\alpha}=\varepsilon^{\ell}$. These two assertions imply that

$$
\delta_{n}^{\left(1-\zeta_{1}\right) \alpha}=\varepsilon^{p \ell}
$$

Since $N r_{\mathbb{B}_{n} / \mathbb{B}_{n-1}}\left(\delta_{n}\right)=1$ and $\left(1-\zeta_{1}\right) \alpha$ is non-zero, the degree of $\varepsilon$ is $p^{n}$ and $N r_{\mathbb{B}_{n} / \mathbb{B}_{n-1}}(\varepsilon)=1$. Hence we have

$$
h t(\varepsilon) \geq \sqrt{p^{n}} \log \left(\frac{p^{\left(p^{n}-1\right) / p^{n}(p-1)}+\sqrt{p^{2\left(p^{n}-1\right) / p^{n}(p-1)}+4}}{2}\right) .
$$

from Lemma $2.5(2)$. 
From (4.3), (4.4) and (4.5), we obtain

$$
\begin{aligned}
p \ell \sqrt{p^{n}} \log & \left(\frac{p^{\left(p^{n}-1\right) / p^{n}(p-1)}+\sqrt{p^{2\left(p^{n}-1\right) / p^{n}(p-1)}+4}}{2}\right) \\
& \leq \sqrt{\frac{2}{\pi}}\left(\frac{d+2}{2} ! \ell^{d-f}\left(\frac{\pi(p-1) p^{p / 2(p-1)} \sqrt{p+1}}{4 \sqrt{3}} \sqrt{p^{n}}\right)^{d}\right)^{1 / d} .
\end{aligned}
$$

This implies

$\ell \leq$

$$
\left.\left(\left(\frac{\sqrt{\pi}(p-1) \sqrt{p+1}}{2 \sqrt{6} p^{(p-2) / 2(p-1)} \log \left(\left(p^{\left(p^{n}-1\right) / p^{n}(p-1)}+\sqrt{\left.p^{2\left(p^{n}-1\right) / p^{n}(p-1)}+4\right)}\right) / 2\right.}\right)\right)^{d} \frac{d+2}{2} !\right)^{1 / f} .
$$

Since $c \geq d$, we can replace $d$ by $c$. Therefore, we have

$$
\begin{aligned}
& \ell \leq \\
& \left(\left(\frac{\sqrt{\pi}(p-1) \sqrt{p+1}}{2 \sqrt{6} p^{(p-2) / 2(p-1)} \log \left(\left(p^{\left(p^{n}-1\right) / p^{n}(p-1)}+\sqrt{\left.p^{2\left(p^{n}-1\right) / p^{n}(p-1)}+4\right) / 2}\right)\right.}\right)^{c} \frac{c+2}{2} !\right)^{1 / f} .
\end{aligned}
$$

From the assumption on $n$, we obtain

$$
\frac{p^{n}-1}{p^{n}(p-1)} \geq \begin{cases}40 / 81, & \text { if } p=3, \\ 31 / 125, & \text { if } p=5, \\ (p+1) / p^{2}, & \text { if } 7 \leq p \leq 19 \\ 1 / p, & \text { if } p \geq 23\end{cases}
$$

This implies $\ell \leq G(p, s, f)$.

\section{Corollary B}

In this section, we show the $\ell$-indivisibility of the class number $h_{n}$ for $p=2$ and $\ell \not \equiv \pm 1(\bmod 64)$.

From Theorem 1.4, we study the cases $\ell \equiv 31(\bmod 64)$ and $\ell \equiv 33$ $(\bmod 64)$.

5.1. $\ell \equiv 31(\bmod 64)$. Let $\ell$ be a prime number with $\ell \equiv 31(\bmod 64)$. Then $f=2, s=6$ and $c=32$. Hence we have

$$
G(2,6,2)=\sqrt{2\left(\frac{\sqrt{\pi}}{\sqrt{2} \log (2+\sqrt{5})}\right)^{32} 17 !}<2777715<10^{9} .
$$


From Theorem 1.4 and Theorem A, $h_{n}$ is indivisible by $\ell$ for every nonnegative integer $n$ if $\ell \equiv 31(\bmod 64)$.

5.2. $\ell \equiv 33(\bmod 64)$. Let $\ell$ be a prime number with $\ell \equiv 33(\bmod 64)$. Then $f=1, s=5$ and $c=16$. Hence we have

$$
G(2,5,1)=2\left(\frac{\sqrt{\pi}}{\sqrt{2} \log (2+\sqrt{5})}\right)^{16} 9 !<75585<10^{9} .
$$

From Theorem 1.4 and Theorem A, $h_{n}$ is indivisible by $\ell$ for every nonnegative integer $n$ if $\ell \equiv 33(\bmod 64)$.

\section{References}

[1] H. BAuer, "Numerische Bestimmung von Klassenzahlen reeller zyklischer Zahlkörper", J. Number Theory 1 (1969), p. 161-162.

[2] H. F. Blichfeldt, "A new principle in the geometry of numbers, with some applications", Trans. Amer. Math. Soc. 15 (1914), no. 3, p. 227-235.

[3] H. Cohn, "A numerical study of Weber's real class number of calculation. I", Numer. Math. 2 (1960), p. 347-362.

[4] - "Proof that Weber's normal units are not perfect powers", Proc. Amer. Math. Soc. 12 (1961), p. 964-966.

[5] T. Fukuda \& K. Komatsu, "Weber's class number problem in the cyclotomic $\mathbb{Z}_{2}$-extension of $\mathbb{Q}$, III", Int. J. Number Theory 7 (2011), no. 6, p. 1627-1635.

[6] L. Gerber, "The orthocentric simplex as an extreme simplex", Pacific J. Math. 56 (1975), no. 1 , p. 97-111.

[7] K. Horie, "Ideal class groups of Iwasawa-theoretical abelian extensions over the rational field", J. London Math. Soc. (2) 66 (2002), no. 2, p. 257-275.

[8] - "The ideal class group of the basic $\mathbf{Z}_{p}$-extension over an imaginary quadratic field", Tohoku Math. J. (2) 57 (2005), no. 3, p. 375-394.

[9] — , "Primary components of the ideal class group of the $\mathbf{Z}_{p}$-extension over $\mathbf{Q}$ for typical inert primes", Proc. Japan Acad. Ser. A Math. Sci. 81 (2005), no. 3, p. 40-43.

[10] - "Certain primary components of the ideal class group of the $\mathbf{Z}_{p}$-extension over the rationals", Tohoku Math. J. (2) 59 (2007), no. 2, p. 259-291.

[11] K. Horie \& M. Horie, "The narrow class groups of some $\mathbb{Z}_{p}$-extensions over the rationals", Acta Arith. 135 (2008), no. 2, p. 159-180.

[12] - "The ideal class group of the $\mathbb{Z}_{23}$-extension over the rational field", Proc. Japan Acad. Ser. A Math. Sci. 85 (2009), no. 10, p. 155-159.

[13] - "The ideal class group of the $\mathbb{Z}_{p}$-extension over the rationals", Tohoku Math. J. (2) 61 (2009), no. 4, p. 551-570.

[14] - "The narrow class groups of the $\mathbb{Z}_{17^{-}}$and $\mathbb{Z}_{19}$-extensions over the rational field", Abh. Math. Semin. Univ. Hambg. 80 (2010), no. 1, p. 47-57.

[15] - "The $l$-class group of the $Z_{p}$-extension over the rational field", J. Math. Soc. Japan 64 (2012), no. 4, p. 1071-1089.

[16] H. IChimura \& S. Nakajima, "On the 2-part of the class numbers of cyclotomic fields of prime power conductors", J. Math. Soc. Japan 64 (2012), no. 1, p. 317-342.

[17] K. IwASAwA, "A note on class numbers of algebraic number fields", Abh. Math. Sem. Univ. Hamburg 20 (1956), p. 257-258.

[18] F. J. VAN DER Linden, "Class number computations of real abelian number fields", Math. Comp. 39 (1982), no. 160, p. 693-707.

[19] J. M. MASLEy, "Class numbers of real cyclic number fields with small conductor", Compositio Math. 37 (1978), no. 3, p. 297-319. 
[20] J. C. Miller, "Class numbers of totally real fields and applications to the Weber class number problem", Acta Arith. 164 (2014), no. 4, p. 381-398.

[21] — "Class numbers in cyclotomic $\mathbb{Z}_{p}$-extensions", J. Number Theory 150 (2015), p. 4773.

[22] T. Morisawa, "Mahler measure of the Horie unit and Weber's class number problem in the cyclotomic $\mathbb{Z}_{3}$-extension of $\mathbb{Q} "$, Acta Arith. 153 (2012), no. 1, p. 35-49.

[23] T. Morisawa \& R. OKAzAKI, "On filtrations of units of Viète field", preprint.

[24] - "Mahler measure and Weber's class number problem in the cyclotomic $\mathbf{Z}_{p}$-extension of $\mathbf{Q}$ for odd prime number p", Tohoku Math. J. (2) 65 (2013), no. 2, p. 253-272.

[25] R. OKAZAKI, "On a lower bound for relative units, Schinzel's lower bound and Weber's class number problem", preprint.

[26] L. C. Washington, "Class numbers and $\mathbf{Z}_{p}$-extensions", Math. Ann. 214 (1975), p. 177-193.

[27] - "The non-p-part of the class number in a cyclotomic $\mathbf{Z}_{p}$-extension", Invent. Math. 49 (1978), no. 1, p. 87-97.

[28] H. WeBer, "Theorie der Abel'schen Zahlkörper", Acta Math. 8 (1886), no. 1, p. 193-263.

[29] - Lehrbuch der Algebra, Braunschweig, Friedrich Vieweg und Sohn, 1895, $\mathrm{xv}+653$ pages.

Takayuki MorisawA

Division of Liberal Arts,

Kogakuin University,

2665-1 Nakano, Hachioji, Tokyo, 192-0015, Japan

E-mail: morisawa@cc.kogakuin.ac.jp

Ryotaro OKAzAKI

Junior Division, College of Arts and Sciences,

The university of Tokyo,

3-8-1, Komaba, Meguro-ku, Tokyo, 153-8902, Japan

E-mail: rokazaki@dd.iij4u.or.jp 